\title{
Experimental observation of switching in ferromagnetic nanoscale double disks
}

\author{
M. Huber, J. Zweck, and D. Weiss \\ Institut für Experimentelle und Angewandte Physik, Universität Regensburg, D-93040 Regensburg, Germany
}

(Received 13 July 2007; revised manuscript received 31 October 2007; published 6 February 2008)

\begin{abstract}
We investigated a system of two overlapping ferromagnetic permalloy disks, called a hysteron. For sufficiently small disk diameters the hysteron contains only one vortex which is displaced off the center of the disk hosting it. By swapping the vortex from one side of the disk to the other via an in-plane magnetic field, the magnetization in the appended disk is reversed. The magnetization reversal process based on these off-center magnetic vortex states was theoretically found to have low switching fields, as magnetization reversal does not require wall nucleation. Here we investigate hysterons experimentally by studying magnetization reversal and configuration by means of Lorentz transmission electron microscopy and electron holography. For the smallest hysterons with individual disk diameters below $\sim 200 \mathrm{~nm}$ we found the peculiar switching scheme suggested recently.
\end{abstract}

DOI: $10.1103 /$ PhysRevB.77.054407

\section{INTRODUCTION}

Ferromagnetic flat disks made of material lacking magnetocrystalline anisotropy possess a vortexlike magnetization configuration if their diameter and thickness is sufficiently small. The vortex consists of a flux-closed arrangement of spins in the plane and a small region with an out-of-plane magnetization in the center. ${ }^{1-3}$ The hysteresis curve measured for an applied in-plane magnetic field shows zero remanence and the magnetization reversal involves nucleation of a vortex, shift of the vortex's center perpendicular to the applied field and annihilation of the vortex. ${ }^{4}$ The reversal of the out of plane center by means of microwave-or ac-current excitation is currently an interesting topic ${ }^{5,6}$ which offers the possibility to use the up and down orientation of vortex's center in memory applications. Here we address experimentally the question whether different modes of switching can be achieved if two ferromagnetic disks overlap forming a peanut shaped structure. Such an arrangement was previously investigated theoretically and called hysteron "because it contains the basic unit for reversal of magnetization by a continuous process of domain wall motion, but without nucleation." ${ }^{, 7} \mathrm{An}$ interesting situation arises if only one of the disks contains a vortex. In this case the symmetry is broken: One disk features a vortex which is displaced from the center of the corresponding disk while the second, partly overlapping disk, has an $s$-state- or $c$-state-like magnetization configuration. Magnetization reversal of such hysterons was studied theoretically as it utilizes Brown's paradox: Since magnetization reversal does not require wall nucleation the required switching fields are low which makes the system suitable as a storage element in magnetic random access memories (MRAM). ${ }^{7,8}$ Corresponding micromagnetic simulations proved the feasibility of the magnetization reversal scheme. For two overlapping and sufficiently small disks, three possible remanent magnetic configurations containing only one magnetic vortex were found using Scheinfein's micromagnetic LLG code. ${ }^{7}$

We reproduced these stable configurations, using the OOMMF code as explained below. Accordant results are depicted in Fig. 1. The magnetization configuration in Fig. 1(a) displays a vortex in the lower half of the left disk, the one in Fig. 1(c) a vortex in the upper half. A third, metastable con-
PACS number(s): 73.43.Jn, 72.25.Dc, 73.43.Qt

figuration is shown in Fig. 1(b). Switching between the state shown in Fig. 1(a) and 1(c) was shown to occur by applying an in-plane switching and bias field in the $x$ and $y$ direction, respectively. ${ }^{7}$ Switching of the vortex position is connected with an almost uniform rotation of the right disk's magnetization. Because this reversal takes place via continuous domain wall motion without nucleation, the switching field is, as pointed out above, low due to Brown's paradox..$^{9}$ The aim of our investigation is to demonstrate such a switching scenario experimentally. Below we describe the fabrication of peanut shaped hysterons and investigate the switching characteristics for different sizes of the double disks. For our smallest devices, we indeed find the suggested switching behavior even without applying a bias field.

\section{METHODS}

Our samples were fabricated on 30-nm-thick $\mathrm{Si}_{3} \mathrm{~N}_{4}$ membranes that were transparent for the electron beam of a transmission electron microscope, enabling both, Lorentz microscopy and electron holography. The hysterons were defined in a two-layer resist consisting of PMMA (polymethyl methacrylate) with high-resolution electron beam lithography (EBL). Afterwards permalloy $\left(\mathrm{Ni}_{81} \mathrm{Fe}_{19}\right)$ with a thickness of $20 \mathrm{~nm}$ was deposited by thermal evaporation at a base pressure below $7 \times 10^{-5} \mathrm{~Pa}$, followed by a lift-off process. We fabricated double disks with disk diameters from $120 \mathrm{~nm}$ up to one $\mu \mathrm{m}$. The disks' overlap was adjusted between 5 and $30 \%$ of the diameter. Below we focus on hysterons with $20 \%$ overlap. Smaller overlap often caused more than one vortex to be inside the devices. A significantly higher overlap, on the other hand, prevented the displaced vortex configuration. An electron micrograph of a completed doubledisk together with a sketch of the sample is shown in Fig. 2.

To probe the magnetization pattern and to investigate switching at room temperature we employed Lorentz transmission electron microscopy (LTEM) using a Tecnai F30 in the Fresnel mode. ${ }^{10}$ In Lorentz microscopy magnetic vortices are displayed as bright or dark spot in the disk centers, depending on the helicity of the in-plane magnetization vortex and the position of the focus plane of the electron beam. ${ }^{1}$ To generate a small in-plane magnetic field we used the objec- 


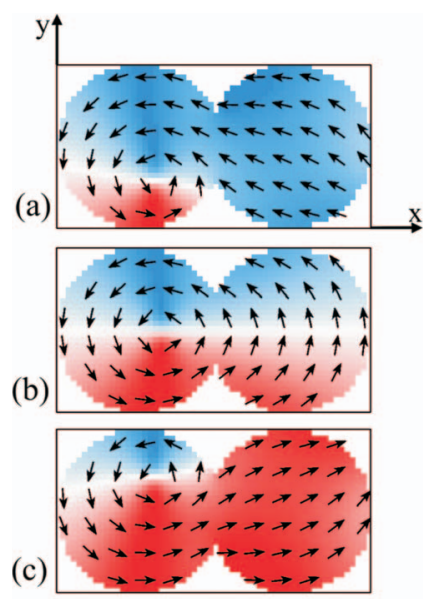

FIG. 1. (Color) Simulated remanent magnetization configurations of hysterons with a disk diameter of $120 \mathrm{~nm}$, overlap $4 \%$ of the disk diameter, and thickness $8 \mathrm{~nm}$. The hysteron with parameters close to the one used by Arrott (Ref. 7) contains one vortex. In a hysteron the vortex is typically located eccentrically in one of the disks, shown in (a) and (c). Depending on the switching conditions also a stable intermediate vortex position (b) can occur. In the simulation a switching field of 50 Oe along the long axis of the hysteron and a bias field of 50 Oe normal to it, both in-plane, were applied.

tive lens of the microscope and tilted the sample holder. Inplane magnetic fields generated this way ranged between $-500 \mathrm{Oe}$ and +500 Oe. The accuracy of the in-plane magnetic field alignment is of order $5^{\circ}$. As the lateral resolution of Lorentz microscopy is typically limited to $\sim 30 \mathrm{~nm}$ we resorted to electron holography with a resolution of about $10 \mathrm{~nm}$ for the smaller devices, also employing the Tecnai F30. In holographic imaging, one detects the phase shifts of an electron wave passing trough the sample. Magnetic phase shifts stem from the Aharonov-Bohm effect and electrostatic phase shifts are due to the electrostatic potential within the specimen. ${ }^{11}$ Our phase images contain both phase contributions. As our samples are relatively flat, the electrostatically induced phase shift appears as a constant background signal only. In electron holography contours of constant $B$ field are represented by the same color. Hence, magnetic vortices appear as colored, ringlike structures where the helicity of the in-plane vortex is given by the color sequence. A corresponding example of a double-disk featuring one vortex in the right disk is shown in Fig. 3.

We compared our experimental results with micromagnetic simulations, using the software package OOMMF (version 1.1 beta). ${ }^{12}$ The hysterons with a thickness of $20 \mathrm{~nm}$, disk diameters ranging from 100 to $500 \mathrm{~nm}$ and an overlap of $20 \%$ were simulated using a size of $2 \mathrm{~nm}$ (4 $\mathrm{nm}$ for hys-

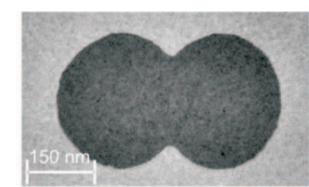

(a)

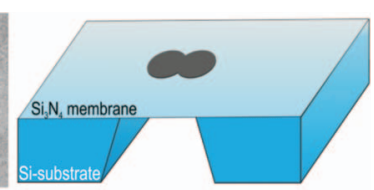

(b)
FIG. 2. (Color) (a) Transmission electron micrograph of a hysteron with a disk diameter of $300 \mathrm{~nm}$, an overlap of $20 \%$, and a thickness of $20 \mathrm{~nm}$ prepared on a $\mathrm{Si}_{3} \mathrm{~N}_{4}$ membrane. (b) Schematic diagram of a patterned hysteron on a $\mathrm{Si}_{3} \mathrm{~N}_{4}$ membrane. (a)

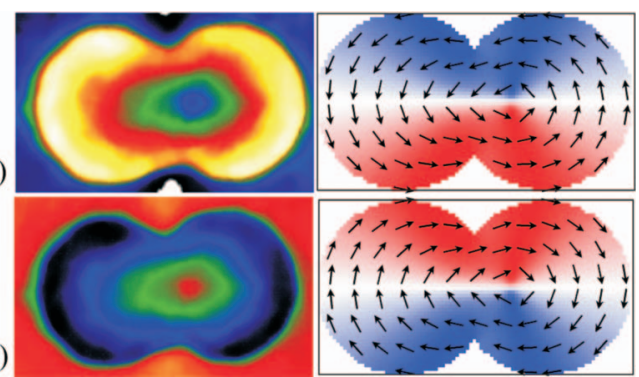

FIG. 3. (Color) (Left): Electron holograms of a single vortex state in a hysteron. Here, the disk diameter was $250 \mathrm{~nm}$. The corresponding micromagnetic simulations are shown in the right hand panel. Images (a) and (b) only differ in the helicity of the vortex: one is clockwise, the other counterclockwise. This is manifested by the false color sequence in the micrographs of the left panel: in (a) the vortex center is blue and evolves over green to red for larger radii while the sequence is inverted in (b).

terons with a disk diameter of $500 \mathrm{~nm}$ ) in a two-dimensional grid. The grid size is smaller than the exchange length of permalloy. We used a saturation field $M_{s}=780 \mathrm{kA} / \mathrm{m}$ and an exchange constant of $A=1.3 \times 10^{-11} \mathrm{~J} / \mathrm{m}$ which are both values typical for permalloy. In the simulations, the magnetic in-plane field was not aligned exactly parallel to an axis to break the symmetry.

\section{RESULTS}

First, we start with displaying typical remanent states after magnetizing hysterons above saturation. The external magnetic field was applied in-plane along the long axis. In Fig. 4 the magnetization patterns of hysterons with individual disk diameters of $300 \mathrm{~nm}$ in Figs. 4(a) and 4(b), $215 \mathrm{~nm}$ in Fig. 4(c), and $150 \mathrm{~nm}$ in Fig. 4(d) are shown. All devices in Fig. 4 show a magnetization pattern with a vortex either in the upper or the lower half of the left disks while the right disk was devoid of a vortex. The states observed in Figs. 4(a)-4(d) are the ones, which form the basis of Arrott's switching proposal. ${ }^{7}$ In Figs. 4(c) and 4(d), the magnetization pattern is compared to corresponding micromagnetic simulations. In the simulations, it was necessary to start with different initial magnetization configurations to obtain all the different remanent states. The curvature of the magnetization in the vortex-free disk gets more developed with increasing disk diameter. For the smallest hysteron, displayed in Fig. 4(d), we obtain essentially the configuration of Fig. 1(a) or 1(c).

The remanent states displayed in Figs. 4(a)-4(d) are only observed in disks with diameters smaller than about $400 \mathrm{~nm}$. However, the one-vortex states are not always the ones observed after saturation. Frequently we observe the magnetization patterns shown in Fig. 5. Figures 5(a) and 5(b) show hysterons with $300 \mathrm{~nm}$ disk diameter where each of the disks accommodates a vortex. Each of the vortices is located near the center of a particular disk, very similar to the situation of two separated disks. The two possible configurations differ in the helicity of the two vortices. In one configuration, they have the same helicity indicated by the two dark spots in Fig. 
(a)

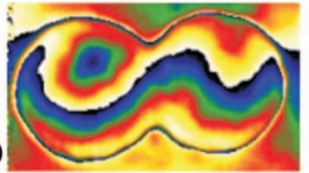

(b)

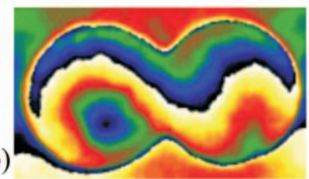

(d)
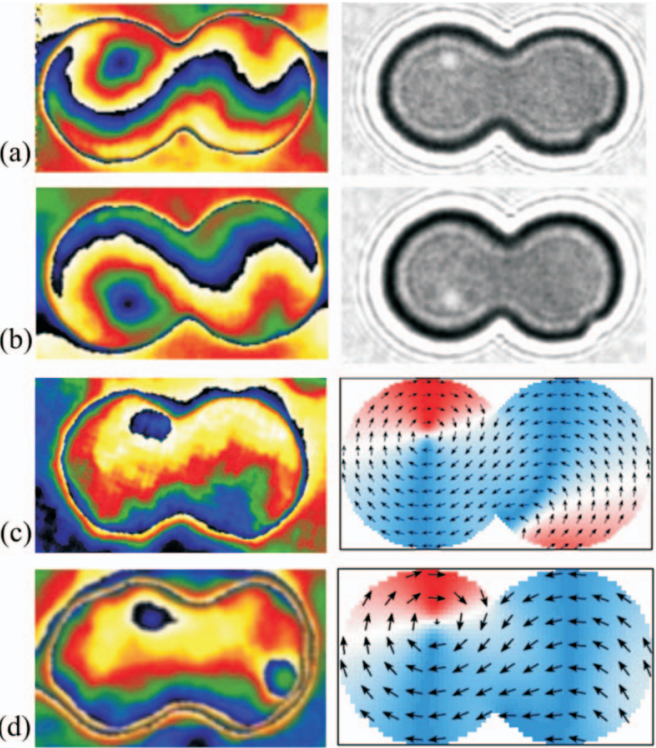

FIG. 4. (Color) Examples of remanent states measured either by electron holography or Lorentz microscopy. (a) Magnetization pattern of two hysterons with $300 \mathrm{~nm}$ individual disk diameter. The left picture was taken with electron holography, the right one with LTEM. Both hysterons show a magnetization pattern with the vortex in the upper half of the left disk. (b) As (a) but with the vortex in the lower half of the left disk. (c) Left: Electron hologram of a double disk with $215 \mathrm{~nm}$ individual disk diameter. Right: Corresponding micromagnetic simulation using OOMMF. (d) Left: Electron hologram of a double disk with $150 \mathrm{~nm}$ individual disk diameter. The vortex is located in the upper half of the left disk. Note that the dot in the right disk is no vortex but an artifact, caused by an electrostatically induced phase shift due to dirt on the sample. Right: Corresponding micromagnetic simulation.

5(a). This configuration results in two walls: one connects the two vortex centers, the other one is normal to this wall and is located at the hysteron's waist. At the intersection of the two walls a Bloch line forms. If the helicity of the two in-plane vortices is opposite [Fig. 5(b)] the magnetization in the center of the hysteron is nearly homogeneous. Our micromagnetic simulations suggest that the two-vortex state is, for the given dimensions, the state with the smallest total energy. For disk diameters smaller than about $400 \mathrm{~nm}$ we sometimes observed an $s$ shaped or $c$ shaped magnetization pattern without any vortex. As this state is energetically unfavorable it occurred only rarely. Another stable configuration we often found in our experiments is an intermediate state with one vortex located on the long axis of the hysteron. For disk diameters smaller than about $140 \mathrm{~nm}$, its total energy is smaller than that of a two-vortex state. This intermediate state, displayed in Fig. 5(c), is the one shown in Fig. 1(b).

To study systematically magnetization reversal of hysterons we first describe a procedure to prepare reliably a remanent eccentric displaced vortex state if, e.g., the original remanent state happens to be a two-vortex state. This was done by applying an in-plane magnetic field in the direction of the hysteron's long axis. Due to small asymmetries of the two disks, the annihilation fields of the vortices in their particular disk are slightly different. Hence, by sweeping to- (a)

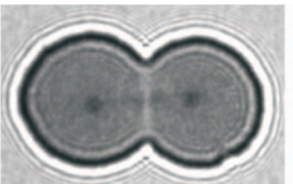

(b)

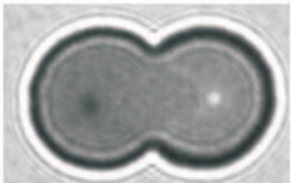

(c)

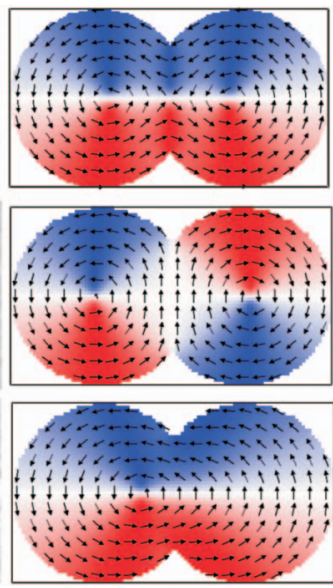

FIG. 5. (Color) Different remanent vortex configurations observable in hysterons with individual disk diameter of $300 \mathrm{~nm}$. Left panels show Lorentz micrographs, right panels the result of corresponding micromagnetic simulations. (a) State with a vortex in each disk of the hysteron. The two in-plane vortices have the same helicity (two dark spots in each of the two disks). (b) Two-vortice state with differing helicity (dark and bright spot). (c) Arrott's intermediate state [see Fig. 1(b)] with one vortex in the left disk.

wards the saturation field one of the vortices usually annihilates before the other. This process is depicted in Fig. 6. By using such an initialization scheme, the desired remanent eccentric one-vortex states have been prepared reliably for disks with diameters smaller than about $350 \mathrm{~nm}$. In case an intermediate vortex state forms in disks with diameters larger

(a)
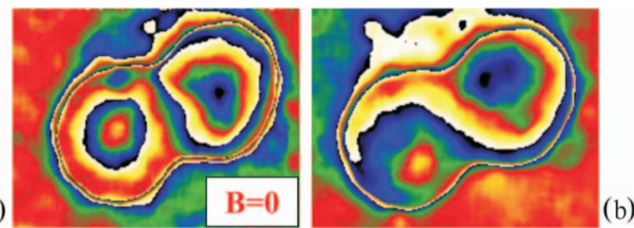

(c)
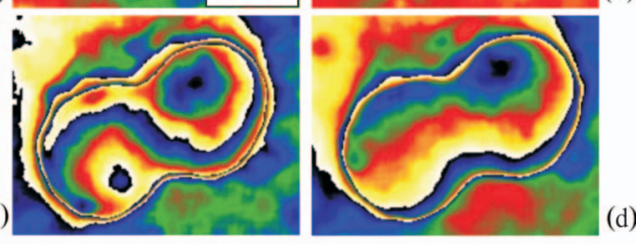

b)
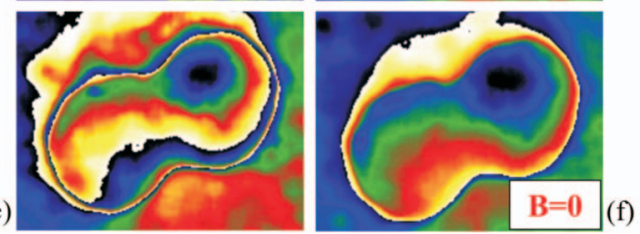

FIG. 6. (Color) Series of electron holograms, which show the preparation of a remanent eccentric vortex state. Here, the individual disk diameter was $200 \mathrm{~nm}$. (a) After saturation a two-vortex state with opposite helicity occurred. In Figs. 6(b)-6(d)the $B$ field, aligned along the long axis of the hystereon was successively ramped up to a maximum value of $\sim 190 \mathrm{Oe}$. Consequently, the vortex centers are shifted in opposite directions towards the edge of the device. Figure 6(d) shows that the lower left vortex has been annihilated first leaving a vortex in the right disk. In Fig. 6(e) the $B$ field is reduced but the vortex remains close to the edge of the hysteron. Figure 6(f) shows the prepared single vortex state after the $B$ field has been switched off. 
(a)

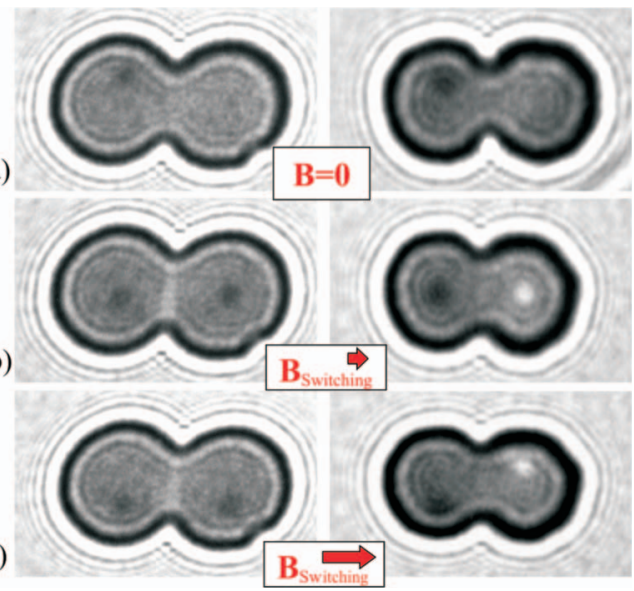

FIG. 7. (Color) Series of LTEM pictures during the switching process of a hysteron with disk diameter $300 \mathrm{~nm}$. The magnetic in-plane field was applied along the hysteron's long axis; no bias field was applied. (a) Starting configuration of two devices with a vortex located near the upper fringe of the left disks, respectively. (b) Application of a switching field applied along the long axis generates a second vortex in the originally vortex-free disks. The vortex's helicity either can be the same (left picture) or oppositely oriented (right picture). These two-vortice states are equilibrium configurations. (c) With increasing field vortices with the same helicity move in the same (left picture), vortices with different helicity in opposite directions (right picture).

than $\sim 200 \mathrm{~nm}$ we magnetized the hysteron so often beyond saturation until the eccentric single vortex state appeared. For disk diameters smaller than $\sim 200 \mathrm{~nm}$, we easily were able to prepare a remanent eccentric vortex this way: we applied a field parallel to the long axis, so that the vortex moved towards to the boundary of the disk. After switching off the field, the magnetization formed the desired eccentric single vortex state. For hysterons with diameters larger than about $400 \mathrm{~nm}$ we never observed the eccentric single vortex state but only the two-vortex state and the intermediate vortex state.

In this section we now describe switching between eccentric single vortex states. In a first set of experiments, the (a)
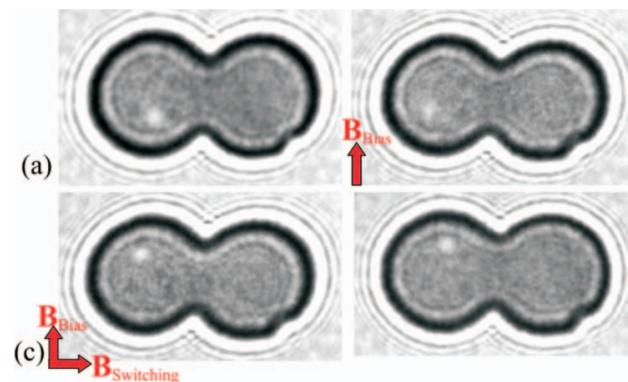

(d)
FIG. 8. (Color) Switching sequence involving a bias field in the $y$ direction. The hysteron has a disk diameter of $300 \mathrm{~nm}$. (a) Initial configuration with a vortex in the lower half of the left disk. (b) A bias field in the $y$ direction of strength $\sim 135$ Oe shifts the vortex, possessing counter clockwise helicity, to the left. (c) In a switching field of $\sim 120$ Oe, applied in the $x$ direction, the vortex jumps into the upper half of the left disk. (d) Remanent configuration after switching. (a)

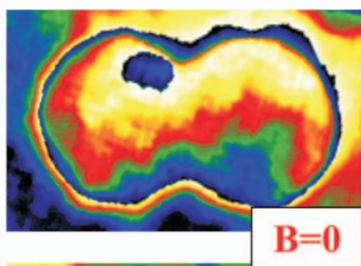

(b)

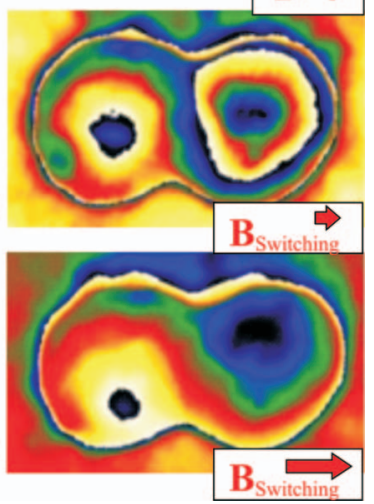

FIG. 9. (Color) Series of electron holograms during the switching process of a hysteron without bias field for smaller hysterons with disk diameter $215 \mathrm{~nm}$. (a) Initial configuration with vortex located in the upper half of the left disk. (b) By applying a switching field parallel to the long axis of the particle a second vortex with opposite helicity forms in the right disk. (c) An increasing switching field shifts both vortices: one towards the lower edge and one to the upper edge, characteristic for the different helicity of both vortices.

switching field was applied in-plane along the hysteron's long axis. Two different samples with a displaced vortex in the upper half of the left disk are displayed in Fig. 7(a). If only a switching field was applied, a second vortex formed in all cases. This effect is shown in Fig. 7(b). Since the total energy of a remanent double-vortex state is, as pointed out

(a)
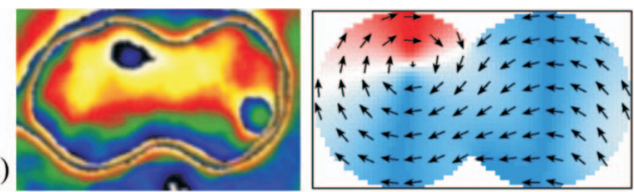

(b)
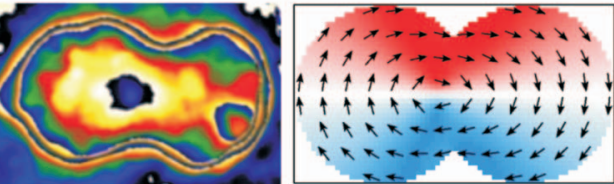

(c)
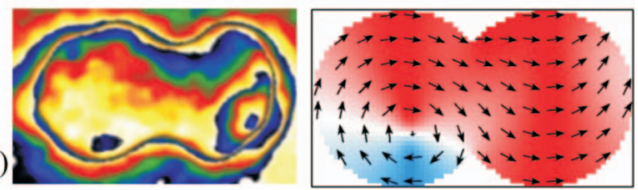

FIG. 10. (Color) Switching of the smallest hysteron with disk diameter of $150 \mathrm{~nm}$. Here, no bias field was applied. The corresponding micromagnetic simulations are shown in the right panel. (a) Initial configuration with a vortex in the upper section of the left disk. The dot like feature in the lower right part of the right disk is an artifact, presumably dirt, not a vortex. (b) If a too low switching field (here $\sim 220 \mathrm{Oe}$ ) is applied along the long axis an intermediate state forms, but (c) for sufficiently strong switching fields (here $\sim 240$ Oe) the vortex jumps to the lower half of the left disk and the magnetization in the vortex free right disk reverses. 
above, slightly lower than that of an eccentric single vortex state (at least for the dimensions shown here), the formation of the double vortex state upon switching is not too surprising. However, the generation of the second vortex can be avoided if the vortex's trajectory is forced to follow the circumference of the disk during switching. By this operation, the curvature of the magnetization in the second, vortex-free disk gets less bent and is hence less susceptible to form a vortex. This can be achieved by a bias field, which is applied normal to the switching field. Therefore, the vortex is shifted perpendicularly to the bias field toward the disk's boundary and avoids the critical area close to the center of the structure. Thus, the formation of a second vortex during the switching process can be prevented. Hence the recipe for a successful switching sequence is: bias field $(\sim 135 \mathrm{Oe})$ on, switching field $(\sim 120$ Oe) on, bias field off and then switching field off.

In our second series of experiments, we therefore applied a switching field parallel to the long axis of the sample and an additional bias field normal to the switching field. The in-plane fields were achieved by tilting the sample holder in two directions. Switching along the lines predicted by Arrott ${ }^{7}$ is displayed in Fig. 8. We start with a remanent vortex state in the lower half of the left disk in Fig. 8(a). With a bias field of $135 \mathrm{Oe}$, the vortex is shifted towards the left edge of the disk, displayed in Fig. 8(b). Switching occurs at a field of 120 Oe with the bias field still applied, see Fig. 8(c). The resulting remanent state after switching off all magnetic fields is shown in Fig. 8(d). We note that a precise adjustment of the switching field strength is mandatory for reliable switching. Otherwise, the vortex gets annihilated (switching field too strong) or the vortex "falls back" and forms the intermediate vortex state (switching field too low).

The switching mechanisms involving a bias field works reliably for hysterons with disk diameters smaller than $\sim 300 \mathrm{~nm}$. In this section, we discuss, as above, switching without a bias field but now for hysterons with smaller disk diameters of $215 \mathrm{~nm}$. The important feature in which larger and smaller hysterons differ is the curvature of the magnetization in the vortex-free disk. For larger disks, the magnetization has an $s$-type shape, clearly visible in the left-handside holograms of Fig. 4(a) and 4(b) while the smaller ones feature a $c$-type configuration [left-hand side of Figs. 4(c) and 4(d)]. Switching of the $215 \mathrm{~nm}$ hysteron without bias field is displayed in Fig. 9. The electron hologram, displayed in Fig. 9(a), shows the initial state with an eccentric vortex in the upper section of the left disk. An in-plane field, applied along the long axis of the hysteron, generates, as for larger hysterons, a second vortex. In contrast to the larger hysterons, the helicity of the second vortex is always opposite to the one of the first vortex. This behavior is ascribed to the $c$-shaped curvature, which determines the helicity of the second vortex. As the helicity of the $c$ state is always opposite to the one of the vortex, displayed, e.g., in the right-hand panels of Figs. 4(c) and 4(d), the two vortices have opposite helicity. Our micromagnetic simulations confirm this. However, as pointed out above, the formation of a second vortex can be avoided if a bias field is applied normal to the switching field.

For our smallest hysterons with individual disk diameters of $150 \mathrm{~nm}$ a different switching scheme is applicable. No bias field is necessary to suppress the formation of a second vortex. Such a switching sequence we show in Fig. 10. In contrast to the larger hysterons the magnetization in the vortex-free disk exhibits a weaker curvature of the $c$ state. The electron hologram of the initial state is shown in Fig. 10(a). A switching field of 240 Oe, applied along the hysteron's long axis swaps the vortex position from the upper to the lower half of the left disk and reverses the magnetization direction in the vortex-free right disk. This is shown in Fig. $10(\mathrm{c})$. This reversal takes place continuously via domain wall motion without nucleation and is characteristic for magnetization reversal via Brown's paradox. Micromagnetic simulations also show that for hysterons with disk dimensions $\sim 150 \mathrm{~nm}$ and smaller no second vortex forms even if no perpendicular bias field is applied. This is due to the nearly homogeneous magnetization pattern of the vortex-free disk. If the switching field is too low, as was the case for the experiment shown in Fig. 10(b), an intermediate state with a vortex close to the center of the hysteron forms.

\section{CONCLUSION}

The experiments described above demonstrate the feasibility of the switching scheme proposed by Arrott. ${ }^{7}$ While larger double disks with a diameter of about $300 \mathrm{~nm}$ often showed two vortices in remanence, the eccentric single vortex state could be reliably prepared by proper initialization. This state formed the initial state for further investigations. Applying a switching field along the long axis of double disks typically in the diameter range between $\sim 200$ and $\sim 300 \mathrm{~nm}$ prompted a second vortex to appear. Application of an additional bias field along the short axis avoids the formation of such a second vortex and the resulting switching process is the one proposed by Arrott. Experiments on our smallest hysterons even showed that the bias field can be omitted, which further simplifies the switching process. The thickness of $20 \mathrm{~nm}$ we used is still rather thick and responsible for the relatively high switching fields of $\sim 240$ Oe for structures with disk diameters of $150 \mathrm{~nm}$ and 120 Oe for hysterons with disk diameters of $300 \mathrm{~nm}$. The thickness of $20 \mathrm{~nm}$ was chosen to give sufficiently high magnetic contrast in experiment. Our micromagnetic simulations show that the field strength for successful switching critically depends on the thickness. For the structure of Fig. 1, which has a disk diameter of $120 \mathrm{~nm}$ and a thickness of $8 \mathrm{~nm}$, we calculate a switching field of $\sim 50$ Oe. For a hysteron with the same disk diameter but a thickness of $20 \mathrm{~nm}$, the switching field increases to $320 \mathrm{Oe}$, a value close to the ones experimentally observed. Therefore, by reducing the thickness of the structure the necessary field strength for switching can be considerably reduced. Since for thinner disks the in-plane demagnetizing fields are smaller, the switching fields get reduced.

\section{ACKNOWLEDGMENTS}

We thank A. Arrott for valuable discussions and the German Science Foundation (DFG) for support via Grant No. SFB 689. 
${ }^{1}$ J. Raabe, R. Pulwey, R. Sattler, T. Schweinböck, J. Zweck, and D. Weiss, J. Appl. Phys. 88, 4437 (2000).

${ }^{2}$ T. Shinjo, T. Okuno, R. Hassdorf, K. Shigeto, and T. Ono, Science 289, 930 (2000).

${ }^{3}$ A. Wachowiak, J. Wiebe, M. Bode, O. Pietzsch, M. Morgenstern, and R. Wiesendanger, Science 298, 577 (2002).

${ }^{4}$ M. Rahm, M. Schneider, J. Biberger, R. Pulwey, J. Zweck, and D. Weiss, Appl. Phys. Lett. 82, 4110 (2003).

${ }^{5}$ B. Van Waeyenberge, A. Puzic, H. Stoll, K. W. Chou, T. Tyliszczak, R. Hertel, M. Fähnle, H. Brückl, K. Rott, G. Reiss, I. Neudecker, D. Weiss, C. H. Back, and G. Schütz, Nature (London) 444, 461 (2006).
${ }^{6}$ K. Yamada, S. Kasai, Y. Nakatani, K. Kobayashi, H. Kohno, A. Thiaville, and T. Ono, Nat. Mater. 6, 270 (2007).

${ }^{7}$ A. S. Arrott, J. Magn. Magn. Mater. 258-259, 25 (2003).

${ }^{8}$ B. S. Kessler and A. S. Arrott, J. Appl. Phys. 97, 10 C502 (2005).

${ }^{9}$ A. Hubert and R. Schäfer, Magnetic Domains (Springer, Berlin, 1998).

${ }^{10}$ J. N. Chapman and M. R. Scheinfein, J. Magn. Magn. Mater. 200, 729 (1999).

${ }^{11}$ A. Tonomura, T. Matsuda, J. Endo, T. Arii, and K. Mihama, Phys. Rev. B 34, 3397 (1986).

${ }^{12} \mathrm{M}$. Donahue and D. Porter, version 1.1 beta, see http:// math.nist.gov/oommf 